\title{
Glycaemic index: claims, consumer attitudes and effects on weight management and postprandial lipaemia
}

\author{
Sarah Hull, Aimee Matthews, Kelly Johnston, Roberta Re and Fiona Angus \\ Leatherhead Food International, Leatherhead, Surrey, UK
}

The glycaemic index (GI) is a measure of the effects that foods have on postprandial blood glucose levels ${ }^{(1)}$. It is an area of important current research because of its potential impact on a variety of health outcomes including disease risk ${ }^{(2)}$ and control of body mass $^{(3)}$. The present communication describes a body of research carried out in order to assess the effects of GI on weight loss and CVD risk factors, in addition to an assessment of nutrition claims made relating to GI and also an evaluation of UK consumer attitudes towards GI.

Study 1 assessed the GI claims made on food and drink packaging worldwide in 2007 by carrying out a global database search and UK supermarket audit. Four qualitative consumer focus groups were held to assess consumer perception of GI and its associated claims. Database searches identified 670 products worldwide that referred to GI, glycaemic load or glycaemic response. Consumer focus groups revealed that the term 'glycaemic index' was not well understood. Claims relating to satiation were also poorly understood, with negative associations made with the term 'fullness'.

In study 2 three commercially-available low-GI diets were included in a randomised controlled four-way parallel intervention study designed to compare their effectiveness. Ethical approval was obtained from South West Surrey Local Research Ethics Committee (reference no. 04/Q1910/67). The diets selected were 'Eat yourself slim and stay slim' (EYS) $)^{(4)}$, 'The glucose revolution: GI plus' (GIP) ${ }^{(5)}$ and 'GI diet' $(\mathrm{GID})^{(6)}$. A control group was given no dietary advice. Fifty-two overweight but otherwise healthy subjects (mean age 40.4 (SE 1.4) years, mean BMI 29.5 (SE 0.6) $\mathrm{kg} / \mathrm{m}^{2}$ ) were recruited and randomly allocated to one of the four diets. The diets were followed for 19 weeks and anthropometric and clinical measurements were made at 0, 6, 14 and 19 weeks. Three $5 \mathrm{~d}$ weighed-food diaries were completed at baseline and weeks 8 and 16. EYS showed an overall $6.6 \%$ decrease in weight, GIP 5.4\% decrease, GID 5.67\% decrease and the control group $1.06 \%$ decrease. These differences were not significant $(P>0.05)$. However, energy intake was significantly reduced in all groups and the loss was sustained for the duration of the study $(P<0.001)$.

Study 3 investigated the effect of GI on postprandial lipaemia in a randomised controlled seven-way cross-over intervention study. Ethical approval was obtained from West Kent Research Ethics Committee (reference no. 07/Q1801/15). Low-, medium- and high-GI test drinks containing $50 \mathrm{~g}$ fructose, sucrose and glucose respectively were consumed in the presence or absence of a fat load (30 $\mathrm{g}$ fat from double cream). Water was included as a negative control. Twelve male subjects (mean age 21.9 (SE 1) years, mean BMI 20.6 $(\mathrm{SE} 0.5) \mathrm{kg} / \mathrm{m}^{2}$ ) were recruited and blood samples were collected at regular intervals for $6 \mathrm{~h}$ after consumption of the test drink. The acute responses of insulin and plasma lipids to the test drink were measured. The medium- and low-GI drinks containing fat significantly increased plasma TAG levels $(P<0.05)$. No significant differences were observed for insulin or other markers of lipid metabolism.

A large number of claims are currently made in relation to GI; however, the consumer research carried out indicated that consumer understanding of the GI concept and potential health benefits was poor. Further consumer education is required in order to promote understanding. Claims relating to appetite control and GI must be carefully phrased to avoid alienating consumers. The weight-loss data demonstrates that a low-GI diet can promote a reduction in energy intake; however, the weight loss achieved on different low-GI diet plans does not vary significantly. More comprehensive research is needed to assess possible benefits in relation to cardiovascular risk factors.

1. Jenkins DJ, Wolever TM, Taylor RH et al. (1981) Am J Clin Nutr 34, 362-366.

2. Barclay AW, Petocz P, McMillan-Price J et al. (2008) Am J Clin Nutr 87, 627-637.

3. Livesey G, Taylor R, Hulshof T \& Howlett J (2008) Am J Clin Nutr 87, Suppl., 258S-268S.

4. Montignac M (1999) Eat Yourself Slim ... and Stay Slim! London: Montignac Publishing UK.

5. Brand-Miller J, Foster-Powell K \& Lintner L (2000) The Glucose Revolution: G.I. Plus. Sydney: Hodder Headline Australia.

6. Worrall-Thompson A, Blades M \& Suthering J (2005) Antony Worrall Thompson's GI Diet. London: Kyle Cathie Ltd. 